\title{
Sums of Products of Cauchy Numbers, Including Generalized Poly-Cauchy Numbers
}

\author{
Takao KOMATSU
}

Wuhan University

(Communicated by S. Nakano)

\begin{abstract}
Many researchers have investigated sums of products of Bernoulli numbers and related types of sums of products. Recently, the concept of poly-Cauchy numbers has been introduced by the author as a generalization of the classical Cauchy number. In this paper, we investigate sums of products of Cauchy numbers including generalized poly-Cauchy numbers. We give a relation among these sums and explicit expressions of sums of any arbitrary products. In addition, we also show the similar results in the case of poly-Cauchy numbers of the second kind and those of mixed ones of both kinds.
\end{abstract}

\section{Introduction}

The Cauchy numbers (of the first kind) $c_{n}$ are defined by the integral of the falling factorial:

$$
c_{n}=\int_{0}^{1} x(x-1) \cdots(x-n+1) d x
$$

([9, Chapter VII]). The numbers $c_{n} / n$ ! are sometimes called the Bernoulli numbers of the second kind (see e.g. [4, 29]). Such numbers have been studied by several authors ([8, 23, 25 , $28,30])$ because they are related to various special combinatorial numbers, including Stirling numbers of both kinds, Bernoulli numbers and harmonic numbers. It is interesting to see that the Cauchy numbers of the first kind $c_{n}$ have the similar properties and expressions to the Bernoulli numbers $B_{n}$. For example, the generating function of the Cauchy numbers of the first kind $c_{n}$ is expressed in terms of the logarithmic function:

$$
\frac{x}{\ln (1+x)}=\sum_{n=0}^{\infty} c_{n} \frac{x^{n}}{n !}
$$

Received October 2, 2013

Mathematics Subject Classification: 05A15, 11B75

This work was supported in part by the grant of Wuhan University and by the grant of Hubei Provincial Experts Program. 
$([9,25])$ and the generating function of Bernoulli numbers $B_{n}$ is expressed in terms of the exponential function:

$$
\frac{x}{e^{x}-1}=\sum_{n=0}^{\infty} B_{n} \frac{x^{n}}{n !}
$$

([9]) or

$$
\frac{x}{1-e^{-x}}=\sum_{n=0}^{\infty} B_{n}^{(1)} \frac{x^{n}}{n !}
$$

([17]). Actually, $B_{n}=B_{n}^{(1)}(n \geq 0)$ except $B_{1}=-1 / 2$ and $B_{1}^{(1)}=1 / 2$. Bernoulli numbers $B_{n}^{(1)}$ can be also written explicitly as

$$
B_{n}^{(1)}=(-1)^{n} \sum_{m=0}^{n}\left\{\begin{array}{l}
n \\
m
\end{array}\right\} \frac{(-1)^{m} m !}{m+1},
$$

where $\left\{\begin{array}{l}n \\ m\end{array}\right\}$ are the Stirling numbers of the second kind, determined by

$$
\left\{\begin{array}{l}
n \\
m
\end{array}\right\}=\frac{1}{m !} \sum_{j=0}^{m}(-1)^{j}\left(\begin{array}{c}
m \\
j
\end{array}\right)(m-j)^{n}
$$

(see e.g. [13]). Recently, Liu, Qi and Ding ([23]) established some recurrence relations about Cauchy numbers of the first kind as analogous results about Bernoulli numbers by Agoh and Dilcher ([3]). Hence, there is the possibility that the similar results hold for Cauchy numbers if some results are established for Bernoulli numbers. For example, Cauchy numbers of the first kind $c_{n}$ can be written explicitly as

$$
c_{n}=(-1)^{n} \sum_{m=0}^{n}\left[\begin{array}{l}
n \\
m
\end{array}\right] \frac{(-1)^{m}}{m+1}
$$

([9, Chapter VII], [25, p.1908]), where $\left[\begin{array}{c}n \\ m\end{array}\right]$ are the (unsigned) Stirling numbers of the first kind, arising as coefficients of the rising factorial

$$
x(x+1) \cdots(x+n-1)=\sum_{m=0}^{n}\left[\begin{array}{l}
n \\
m
\end{array}\right] x^{m}
$$

(see e.g. [13]).

The following identity on sums of two products of Bernoulli numbers is known as Euler's formula:

$$
\sum_{i=0}^{n}\left(\begin{array}{l}
n \\
i
\end{array}\right) B_{i} B_{n-i}=-n B_{n-1}-(n-1) B_{n} \quad(n \geq 0) .
$$


The corresponding formula for Cauchy numbers was discovered in [30]:

$$
\sum_{i=0}^{n}\left(\begin{array}{l}
n \\
i
\end{array}\right) c_{i} c_{n-i}=-n(n-2) c_{n-1}-(n-1) c_{n} \quad(n \geq 0) .
$$

Many generalizations of (3) have been considered. For example, Dilcher [11] gave closed formulae of sums of $N$ products of Bernoulli numbers for any integer $N \geq 1$. Eie [12] considered the sums of products of Shintani generalized Bernoulli numbers. Other types of sums of products have been also studied (e.g. [1, 2, 6, 15, 19, 24, 26, 27]). Recently, Kamano [14] investigated the following types of sums of products:

$$
S_{m}^{(k)}(n):=\sum_{\substack{i_{1}+\cdots, i_{m}=n \\
i_{1}, \ldots, i_{m} \geq 0}}\left(\begin{array}{c}
n \\
i_{1}, \ldots, i_{m}
\end{array}\right) \underbrace{B_{i_{1}} \cdots B_{i_{m-1}}}_{m-1} B_{i_{m}}^{(k)} \quad(m \geq 1, n \geq 0),
$$

where Bernoulli numbers $B_{n}$ are defined by the generating function (1) and $B_{n}^{(k)}(n \geq 0$, $k \geq 1$ ) are poly-Bernoulli numbers defined by the generating function

$$
\frac{\operatorname{Li}_{k}\left(1-e^{-x}\right)}{1-e^{-x}}=\sum_{n=0}^{\infty} B_{n}^{(k)} \frac{x^{n}}{n !}
$$

where

$$
\operatorname{Li}_{k}(z)=\sum_{m=1}^{\infty} \frac{z^{m}}{m^{k}}
$$

is the $k$-th polylogarithm function. When $k=1, B_{n}^{(1)}=(-1)^{n} B_{n}$ is the classical Bernoulli number with $B_{1}^{(1)}=1 / 2$ as seen in (1) and (2). Kamano also obtained explicit formulae of $S_{m}^{(k)}$ for $m=2$ and 3. Putting $k=1$ in his result $S_{2}^{(k)}=B_{n}^{(1)}-n \sum_{j=1}^{k} B_{n}^{(j)}$, Euler's formula (3) is entailed.

On the other hand, we [22] introduced generalized poly-Cauchy numbers (of the first kind) $c_{n, a}^{(k)}$ as a generalization of the Cauchy numbers and an analogue of the poly-Bernoulli numbers by the following.

$$
\begin{gathered}
c_{n, a}^{(k)}=\int_{0}^{l_{1}} \cdots \int_{0}^{l_{k}}\left(x_{1} x_{2} \cdots x_{k}\right)^{a}\left(x_{1} x_{2} \cdots x_{k}-1\right) \\
\cdots\left(x_{1} x_{2} \cdots x_{k}-(n-1)\right) d x_{1} d x_{2} \cdots d x_{k} .
\end{gathered}
$$

In addition, the generating function of generalized poly-Cauchy numbers is given by

$$
\operatorname{Lif}_{k}(\ln (1+x) ; a)=\sum_{n=0}^{\infty} c_{n, a}^{(k)} \frac{x^{n}}{n !}
$$


where

$$
\operatorname{Lif}_{k}(z ; a):=\sum_{m=0}^{\infty} \frac{z^{m}}{m !(m+a)^{k}} \quad(a>0) .
$$

If $a=1$, then $\operatorname{Lif}_{k}(z ; 1)=\operatorname{Lif}_{k}(z)$ is the $k$-th polylogarithm factorial function, which is also introduced by the author $[18,16]$. If $a=1$, then $c_{n, 1}^{(k)}=c_{n}^{(k)}$ is the poly-Cauchy number (of the first kind), introduced in [18]. If $k=a=1$, then $c_{n, 1}^{(1)}=c_{n}$ is the classical Cauchy number.

In the definition of $c_{n, a}^{(k)}$, the integer $k$ should be positive. However, in the generating function of $c_{n, a}^{(k)}$, the integer $k$ may not be restricted to be positive but extended to zero or negative too.

In this paper, as an analogue of (5), we investigate about sums of products of Cauchy numbers including generalized poly-Cauchy numbers:

$$
T_{m, a}^{(k)}(n):=\sum_{\substack{i_{1}+\cdots+i_{m}=n \\
i_{1}, \ldots, i_{m} \geq 0}}\left(\begin{array}{c}
n \\
i_{1}, \ldots, i_{m}
\end{array}\right) \underbrace{c_{i_{1}} \cdots c_{i_{m-1}}}_{m-1} c_{i_{m}, a}^{(k)} \quad(m \geq 1, n \geq 0) .
$$

A relation among these sums and explicit expressions of sums of two and three products are also given. By the identity $T_{2}^{(k)}=T_{2,1}^{(k)}$, we get the corresponding formula (4) of Cauchy numbers to Euler's formula. If $a=1$, the results in this paper are reduced to those in [21]. Not only so. Kamano [14] mentioned that explicit formulae of $S_{m}^{(k)}$ for $m \geq 4$ seemed to be complicated to describe. We obtain explicit formulae of $T_{m}^{(k)}$ for any $m \geq 2$. In addition, we give analogous formulae for poly-Cauchy numbers of the second kind and for mixed formulae of both kinds of Cauchy numbers.

\section{Main results}

It is shown [14] that

$$
\sum_{l=0}^{m}(-1)^{m-l}\left[\begin{array}{c}
m+1 \\
l+1
\end{array}\right] S_{m+1}^{(k-l)}(n)= \begin{cases}\frac{n !}{(n-m) !} \sum_{l=0}^{m}\left[\begin{array}{c}
m \\
l
\end{array}\right] B_{n-m+l}^{(k)}, & (n \geq m) \\
0, & (0 \leq n \leq m-1) .\end{cases}
$$

We consider an extended analogous type of sums of products of Cauchy numbers including poly-Cauchy numbers $T_{m, a}^{(k)}$ and obtain the following relation: 
THEOREM 1. For an integer $k$ and a non-negative integer $m$, we have

$$
\sum_{l=0}^{m}(-1)^{m-l} f_{m, l} T_{m+1, a}^{(k-l)}(n)= \begin{cases}\sum_{l=0}^{m} \sum_{i=0}^{n-m} \frac{n !}{i !}\left(\begin{array}{c}
l \\
n-m-i
\end{array}\right)\left\{\begin{array}{c}
m \\
l
\end{array}\right\} c_{l+i, a}^{(k)} & (n \geq m) \\
0 & (0 \leq n \leq m-1)\end{cases}
$$

where $f_{m, l}(l=0,1, \ldots, m)$ are coefficients, yielding

$$
\begin{aligned}
(x-a)(x-(a+1)) \cdots & (x-(a+n-1)) \\
= & f_{n, n} x^{n}-f_{n, n-1} x^{n-1}+f_{n, n-2} x^{n-2}+\cdots+(-1)^{n} f_{n, 0} .
\end{aligned}
$$

REMARK. By the definition

$$
\begin{aligned}
f_{m, 0} & =a(a+1) \cdots(a+m-1)=\sum_{i=0}^{m}\left[\begin{array}{c}
m \\
i
\end{array}\right] a^{i}, \\
f_{m, l} & =\frac{1}{l !} \frac{d^{l}}{d a^{l}} f_{m, 0} \\
& =\sum_{i=0}^{m-l}\left(\begin{array}{c}
i+l \\
l
\end{array}\right)\left[\begin{array}{c}
m \\
i+l
\end{array}\right] a^{i} \quad(l=1,2, \ldots, m) .
\end{aligned}
$$

If $a=1$, then

$$
f_{m, l}=\left[\begin{array}{c}
m+1 \\
l+1
\end{array}\right]
$$

Note that the generating function of $T_{m, a}^{(k)}$ is given by

$$
\left(\frac{x}{\ln (1+x)}\right)^{m-1} G_{k, a}(x)=\sum_{n=0}^{\infty} T_{m, a}^{(k)}(n) \frac{x^{n}}{n !},
$$

where

$$
\begin{aligned}
G_{k, a}(x): & =\operatorname{Lif}_{k}(\ln (1+x) ; a)=\sum_{n=0}^{\infty} c_{n, a}^{(k)} \frac{x^{n}}{n !} \\
& =\sum_{m=0}^{\infty} \frac{(\ln (1+x))^{m}}{m !(m+a)^{k}} .
\end{aligned}
$$

Since

$$
x^{m} \frac{d^{l}}{d x^{l}} G_{k, a}(x)=\sum_{i=0}^{\infty} c_{l+i, a}^{(k)} \frac{x^{m+i}}{i !} \quad(m, l \geq 0, k \geq 1)
$$


the coefficient of $x^{n}$ in

$$
x^{m} \frac{d^{l}}{d x^{l}} G_{k, a}(x)
$$

is equal to

$$
\begin{cases}\frac{c_{n-m+l, a}^{(k)}}{(n-m) !} & (n \geq m) \\ 0 & (0 \leq n \leq m-1) .\end{cases}
$$

We need the following Lemma in order to prove Theorem 1.

LEMMA 1. For an integer $k$, a positive integer $m$ and a nonzero real number $a$, we have

$$
\begin{array}{r}
\left(\left\{\begin{array}{l}
m \\
m
\end{array}\right\} \frac{d^{m}}{d x^{m}}+\left\{\begin{array}{c}
m \\
m-1
\end{array}\right\} \frac{1}{1+x} \frac{d^{m-1}}{d x^{m-1}}+\cdots+\left\{\begin{array}{c}
m \\
1
\end{array}\right\} \frac{1}{(1+x)^{m-1}} \frac{d}{d x}\right) G_{k, a}(x) \\
=\frac{1}{(1+x)^{m}(\ln (1+x))^{m}} \sum_{l=0}^{m}(-1)^{m-l} f_{m, l} G_{k-l, a}(x),
\end{array}
$$

where $f_{m, l}(l=0,1, \ldots, m)$ are defined in (9).

Proof OF LEMMA 1. Since

$$
\begin{aligned}
\frac{d}{d x} \operatorname{Lif}_{k}(x ; a) & =\frac{1}{x} \sum_{m=0}^{\infty} \frac{m x^{m}}{m !(m+a)^{k}} \\
& =\frac{1}{x} \sum_{m=0}^{\infty}\left(\frac{x^{m}}{m !(m+a)^{k-1}}-a \frac{x^{m}}{m !(m+a)^{k}}\right)=\frac{\operatorname{Lif}_{k-1}(x ; a)-a \operatorname{Lif}_{k}(x ; a)}{x},
\end{aligned}
$$

we have

$$
\frac{d}{d x} G_{k, a}(x)=\frac{G_{k-1, a}(x)-a G_{k, a}(x)}{(1+x) \ln (1+x)} .
$$

By induction, we can show that for $m \geq 1$

$$
\frac{d^{m}}{d x^{m}} G_{k, a}(x)=\sum_{\nu=1}^{m} \frac{(-1)^{m-v}}{(1+x)^{m}(\ln (1+x))^{v}}\left[\begin{array}{c}
m \\
v
\end{array}\right] g_{v+1, a}(x),
$$

where

$$
g_{v+1, a}(x):=\sum_{l=0}^{v}(-1)^{\nu-l} f_{v, l} G_{k-l}(x) \quad(v=1,2, \ldots, m) .
$$


Thus, by using the inversion relationship

$$
\sum_{j=v}^{m}(-1)^{j-v}\left\{\begin{array}{l}
m \\
j
\end{array}\right\}\left[\begin{array}{l}
j \\
v
\end{array}\right]= \begin{cases}1 & (v=m) ; \\
0 & (v=1,2, \ldots, m-1)\end{cases}
$$

(see e.g. [13, Chapter 6]), the left-hand side of the identity in above Lemma is equal to

$$
\begin{aligned}
& \sum_{j=1}^{m}\left\{\begin{array}{c}
m \\
j
\end{array}\right\} \frac{1}{(1+x)^{m-j}} \sum_{\nu=1}^{j} \frac{(-1)^{j-v}}{(1+x)^{j}(\ln (1+x))^{v}}\left[\begin{array}{c}
j \\
v
\end{array}\right] g_{v+1, a}(x) \\
& =\frac{1}{(1+x)^{m}} \sum_{\nu=1}^{m} \frac{g_{v+1, a}(x)}{(\ln (1+x))^{v}} \sum_{j=v}^{m}(-1)^{j-v}\left\{\begin{array}{c}
m \\
j
\end{array}\right\}\left[\begin{array}{c}
j \\
v
\end{array}\right] \\
& =\frac{g_{m+1, a}(x)}{(1+x)^{m}(\ln (1+x))^{m}},
\end{aligned}
$$

which is the right-hand side of the desired identity.

Now, by the generating function (10), the identity (11) and Lemma 1,

$$
\begin{aligned}
& \sum_{n=0}^{\infty}\left(\sum_{l=0}^{m}(-1)^{m-l} f_{m, l} T_{m+1, a}^{(k-l)}(n)\right) \frac{x^{n}}{n !} \\
&=\left(\frac{x}{\ln (1+x)}\right)^{m} \sum_{l=0}^{m}(-1)^{m-l} f_{m, l} G_{k-l, a}(x) \\
&=x^{m}\left((1+x)^{m}\left\{\begin{array}{l}
m \\
m
\end{array}\right\} \frac{d^{m}}{d x^{m}}+(1+x)^{m-1}\left\{\begin{array}{c}
m \\
m-1
\end{array}\right\} \frac{d^{m-1}}{d x^{m-1}}\right. \\
&\left.+\cdots+(1+x)\left\{\begin{array}{c}
m \\
1
\end{array}\right\} \frac{d}{d x}\right) G_{k, a}(x) \\
&= \sum_{l=0}^{m}(1+x)^{l}\left\{\begin{array}{c}
m \\
l
\end{array}\right\} \sum_{i=0}^{\infty} c_{l+i, a}^{(k)} \frac{x^{m+i}}{i !} \\
&= \sum_{l=0}^{m} \sum_{\kappa=0}^{l}\left(\begin{array}{c}
l \\
\kappa
\end{array}\right) x^{\kappa}\left\{\begin{array}{c}
m \\
l
\end{array}\right\} \sum_{i=0}^{\infty} c_{l+i, a}^{(k)} \frac{x^{m+i}}{i !} \\
&= \sum_{l=0}^{m} \sum_{n=0}^{\infty} \sum_{i=0}^{n}\left(\begin{array}{c}
l \\
n-i
\end{array}\right)\left\{\begin{array}{c}
m \\
l
\end{array}\right\} \frac{c_{l+i, a}^{(k)}}{i !} x^{m+n} \\
&= \sum_{n=m}^{\infty}\left(\sum_{l=0}^{m} \sum_{i=0}^{n-m} \frac{n !}{i !}\left(\begin{array}{c}
l \\
n-m-i
\end{array}\right)\left\{\begin{array}{c}
m \\
l
\end{array}\right\} c_{l+i, a}^{(k)}\right) \frac{x^{n}}{n !} .
\end{aligned}
$$


Note that $\left(\begin{array}{l}l \\ v\end{array}\right)=0(l<v)$ and $\left\{\begin{array}{c}m \\ 0\end{array}\right\}=0(m \geq 1)$. Therefore,

$$
\sum_{l=0}^{m}(-1)^{m-l} f_{m, l} T_{m+1, a}^{(k-l)}(n)= \begin{cases}\sum_{l=0}^{m} \sum_{i=0}^{n-m} \frac{n !}{i !}\left(\begin{array}{c}
l \\
n-m-i
\end{array}\right)\left\{\begin{array}{c}
m \\
l
\end{array}\right\} c_{l+i, a}^{(k)} & (n \geq m) ; \\
0 & (0 \leq n \leq m-1) .\end{cases}
$$

If we put $m=1$ in Theorem 1 , by $f_{1,0}=a$ and $f_{1,1}=1$, we get an analogous formula to Euler's identity (3) for sums of products of a Cauchy number and a poly-Cauchy number.

COROLlary 1.

$$
\sum_{i=0}^{n}\left(\begin{array}{l}
n \\
i
\end{array}\right) c_{i}\left(c_{n-i, a}^{(k-1)}-a c_{n-i, a}^{(k)}\right)=n(n-1) c_{n-1, a}^{(k)}+n c_{n, a}^{(k)} \quad(n \geq 0) .
$$

2.1. Explicit formula for $T_{2, a}^{(k)}(n)$. Theorem 1 gives only a relation among sums of products $T_{m, a}^{(k)}(n)$. For $m=2$, an explicit formula for

$$
T_{2, a}^{(k)}(n)=\sum_{i=0}^{n}\left(\begin{array}{l}
n \\
i
\end{array}\right) c_{i} c_{n-i, a}^{(k)}
$$

can be given. This is an extension of [20, Theorem 4].

THEOREM 2. For integers $n \geq 0$ and $k \geq 1$ and a real number $a>0$ we have

$$
\begin{aligned}
T_{2, a}^{(0)}(n) & =c_{n}+n c_{n-1}, \\
T_{2, a}^{(k)}(n) & =\frac{T_{2, a}^{(0)}(n)}{a^{k}}-n \sum_{j=1}^{k} \frac{c_{n, a}^{(j)}+(n-1) c_{n-1, a}^{(j)}}{a^{k-j+1}}, \\
T_{2, a}^{(-k)}(n) & =a^{k} T_{2, a}^{(0)}(n)+n \sum_{j=0}^{k-1} a^{k-j-1}\left(c_{n, a}^{(-j)}+(n-1) c_{n-1, a}^{(-j)}\right) .
\end{aligned}
$$

PROOF.

$$
\begin{aligned}
\sum_{n=0}^{\infty} T_{2, a}^{(0)}(n) \frac{x^{n}}{n !} & =\frac{x}{\ln (1+x)} \operatorname{Lif}_{0}(\ln (1+x) ; a)=\frac{(1+x) x}{\ln (1+x)} \\
& =\frac{x}{\ln (1+x)}+\frac{x^{2}}{\ln (1+x)}
\end{aligned}
$$

Since

$$
\frac{x^{2}}{\ln (1+x)}=x \frac{x}{\ln (1+x)}=x \sum_{n=0}^{\infty} c_{n}^{(k)} \frac{x^{n}}{n !}
$$




$$
=\sum_{n=0}^{\infty}(n+1) c_{n} \frac{x^{n+1}}{(n+1) !}=\sum_{n=0}^{\infty} n c_{n-1} \frac{x^{n}}{n !},
$$

we have

$$
\sum_{n=0}^{\infty} T_{2, a}^{(0)}(n) \frac{x^{n}}{n !}=\sum_{n=0}^{\infty}\left(c_{n}+n c_{n-1}\right) \frac{x^{n}}{n !} .
$$

Next, by (12) and $G_{0, a}(x)=1+x$ we have

$$
\begin{aligned}
\sum_{j=1}^{k} \frac{1}{a^{k-j}}(1+x) \ln (1+x) \frac{d}{d x} G_{j, a}(x) & =\sum_{j=1}^{k}\left(\frac{G_{j-1, a}(x)}{a^{k-j}}-\frac{G_{j, a}(x)}{a^{k-j-1}}\right) \\
& =\frac{1+x}{a^{k-1}}-a G_{k, a}(x) .
\end{aligned}
$$

Hence,

$$
\begin{aligned}
\sum_{n=0}^{\infty} T_{2, a}^{(k)}(n) \frac{x^{n}}{n !} & =\frac{x}{\ln (1+x)} G_{k, a}(x) \\
& =\frac{1}{a^{k}} \frac{x(1+x)}{\ln (1+x)}-x(1+x) \sum_{j=1}^{k} \frac{1}{a^{k-j+1}} \frac{d}{d x} G_{j, a}(x) \\
& =\frac{1}{a^{k}} \sum_{n=0}^{\infty} T_{2, a}^{(0)}(n) \frac{x^{n}}{n !}-x(1+x) \sum_{j=1}^{k} \frac{1}{a^{k-j+1}} \sum_{n=0}^{\infty} c_{n+1, a}^{(j)} \frac{x^{n}}{n !} \\
& =\frac{1}{a^{k}} \sum_{n=0}^{\infty} T_{2, a}^{(0)}(n) \frac{x^{n}}{n !}-\sum_{n=0}^{\infty} n \sum_{j=1}^{k} \frac{1}{a^{k-j+1}}\left(c_{n, a}^{(j)}+(n-1) c_{n-1, a}^{(j)}\right) \frac{x^{n}}{n !} .
\end{aligned}
$$

Therefore, we get the identity (14).

Finally, by

$$
(1+x) \ln (1+x) \frac{d}{d x} G_{-k}=G_{-k-1}-a G_{-k},
$$

we have

$$
\begin{aligned}
\sum_{j=0}^{k-1} a^{k-j-1}(1+x) \ln (1+x) \frac{d}{d x} G_{-j, a}(x) & =\sum_{j=0}^{k-1}\left(a^{k-j-1} G_{-j-1, a}(x)-a^{k-j} G_{-j, a}(x)\right) \\
& =G_{-k, a}(x)-a^{k}(1+x) .
\end{aligned}
$$

Hence,

$$
\sum_{n=0}^{\infty} T_{2, a}^{(-k)}(n) \frac{x^{n}}{n !}=\frac{x}{\ln (1+x)} G_{-k, a}(x)
$$




$$
\begin{aligned}
& =a^{k} \frac{x(1+x)}{\ln (1+x)}+x(1+x) \sum_{j=0}^{k-1} a^{k-j-1} \frac{d}{d x} G_{-j, a}(x) \\
& =a^{k} \sum_{n=0}^{\infty} T_{2, a}^{(0)}(n) \frac{x^{n}}{n !}+x(1+x) \sum_{j=0}^{k-1} a^{k-j-1} \sum_{n=0}^{\infty} c_{n+1, a}^{(-j)} \frac{x^{n}}{n !} \\
& =a^{k} \sum_{n=0}^{\infty} T_{2, a}^{(0)}(n) \frac{x^{n}}{n !}+\sum_{n=0}^{\infty} n \sum_{j=0}^{k-1} a^{k-j-1}\left(c_{n, a}^{(-j)}+(n-1) c_{n-1, a}^{(-j)}\right) \frac{x^{n}}{n !} .
\end{aligned}
$$

Therefore, we get the identity (15).

Putting $k=1$ in (14), we have the following identity. This is also an analogous formula to Euler's identity (3). If $a=1$, then this is reduced to (4).

COROLlary 2.

$$
a \sum_{i=0}^{n}\left(\begin{array}{l}
n \\
i
\end{array}\right) c_{i} c_{n-i, a}=c_{n}+n c_{n-1}-n c_{n, a}-n(n-1) c_{n-1, a} \quad(n \geq 0) .
$$

ProOF. If $k=1$ in (14), we have

$$
T_{2, a}^{(1)}(n)=\frac{T_{2, a}^{(0)}(n)}{a}-\frac{n\left(c_{n, a}+(n-1) c_{n-1, a}\right)}{a} .
$$

Hence,

$$
\sum_{i=0}^{n}\left(\begin{array}{l}
n \\
i
\end{array}\right) c_{i} c_{n-i, a}=\frac{c_{n}+n c_{n-1}}{a}-\frac{n\left(c_{n, a}+(n-1) c_{n-1, a}\right)}{a}
$$

2.2. Explicit formulae for $T_{3, a}^{(k)}(n)$. For $m=3$, an explicit formula for

$$
T_{3, a}^{(k)}(n)=\sum_{i=0}^{n} \sum_{j=0}^{n-i} \frac{n !}{i ! j !(n-i-j) !} c_{i} c_{j} c_{n-i-j, a}^{(k)}
$$

can be also given. This is an extension of [20, Theorem 6].

THEOREM 3. For integers $n \geq 0$ and $k \geq 1$ and a real number $a>0$, we have

$$
\begin{aligned}
& T_{3, a}^{(0)}(n)=-(n-1) c_{n}-2 n(n-2) c_{n-1}-n(n-1)(n-3) c_{n-2}, \\
& T_{3, a}^{(k)}(n)=\frac{T_{3, a}^{(0)}(n)}{a^{k}}-n\left(\frac{1}{a^{k}}-\frac{1}{(a+1)^{k}}\right) T_{2, a}^{(0)}(n-1)
\end{aligned}
$$




$$
+n(n-1) \sum_{j=1}^{k}\left(\frac{1}{a^{k-j+1}}-\frac{1}{(a+1)^{k-j+1}}\right)\left(c_{n, a}^{(j)}+(2 n-3) c_{n-1, a}^{(j)}+(n-2)^{2} c_{n-2, a}^{(j)}\right),
$$

$$
\begin{aligned}
T_{3, a}^{(-k)}(n)= & a^{k} T_{3, a}^{(0)}(n)+n\left((a+1)^{k}-a^{k}\right) T_{2, a}^{(0)}(n-1) \\
& +n(n-1) \sum_{j=0}^{k-2}\left((a+1)^{k-j-1}-a^{k-j-1}\right)\left(c_{n, a}^{(-j)}+(2 n-3) c_{n-1, a}^{(-j)}+(n-2)^{2} c_{n-2, a}^{(-j)}\right) .
\end{aligned}
$$

Proof.

$$
\begin{aligned}
\sum_{n=0}^{\infty} T_{3, a}^{(0)}(n) \frac{x^{n}}{n !} & =\frac{x^{2}(1+x)}{(\ln (1+x))^{2}}=(1+x) \sum_{n=0}^{\infty} T_{2,1}^{(1)}(n) \frac{x^{n}}{n !} \\
& =\sum_{n=0}^{\infty}\left(T_{2,1}^{(1)}(n)+n T_{2,1}^{(1)}(n-1)\right) \frac{x^{n}}{n !} .
\end{aligned}
$$

Since $T_{2,1}^{(1)}(n)=-(n-1) c_{n}-n(n-2) c_{n-1}$ by Corollary 2 ,

$$
\begin{aligned}
T_{3, a}^{(0)}(n)= & T_{2,1}^{(1)}(n)+n T_{2,1}^{(1)}(n-1) \\
= & -(n-1) c_{n}-n(n-2) c_{n-1} \\
& \quad+n\left(-(n-2) c_{n-1}-(n-1)(n-3) c_{n-2}\right) \\
= & -(n-1) c_{n}-2 n(n-2) c_{n-1}-n(n-1)(n-3) c_{n-2},
\end{aligned}
$$

which is the identity (16).

Next, by Lemma 1 with $m=2$ and $f_{2,0}=a^{2}+a, f_{2,1}=2 a+1$ and $f_{2,2}=1$

$$
\begin{aligned}
& \left(\frac{d^{2}}{d x^{2}}+\frac{1}{1+x} \frac{d}{d x}\right) G_{k, a}(x)=\frac{\left(\left(a^{2}+a\right) G_{k, a}(x)-(2 a+1) G_{k-1, a}(x)+G_{k-2, a}(x)\right)}{(1+x)^{2}(\ln (1+x))^{2}} \\
& =\frac{(a+1)\left(a G_{k, a}(x)-G_{k-1, a}(x)\right)-\left(a G_{k-1, a}(x)-G_{k-2, a}(x)\right)}{(1+x)^{2}(\ln (1+x))^{2}} .
\end{aligned}
$$

Thus,

$$
\begin{aligned}
& (1+x)^{2}(\ln (1+x))^{2}\left(\frac{d^{2}}{d x^{2}}+\frac{1}{1+x} \frac{d}{d x}\right) \sum_{j=1}^{k} \frac{G_{j, a}(x)}{(a+1)^{k-j+1}} \\
& =\sum_{j=1}^{k}\left(\frac{a G_{j, a}(x)-G_{j-1, a}(x)}{(a+1)^{k-j}}-\frac{a G_{j-1, a}(x)-G_{j-2, a}(x)}{(a+1)^{k-j+1}}\right) \\
& =a G_{k, a}(x)-G_{k-1, a}(x)-\frac{a G_{0, a}(x)-G_{-1, a}(x)}{(a+1)^{k}} .
\end{aligned}
$$


Hence,

$$
\begin{aligned}
& G_{k, a}(x)-\frac{G_{k-1, a}(x)}{a} \\
& =\frac{a G_{0, a}(x)-G_{-1, a}(x)}{a(a+1)^{k}}+(1+x)^{2}(\ln (1+x))^{2}\left(\frac{d^{2}}{d x^{2}}+\frac{1}{1+x} \frac{d}{d x}\right) \sum_{j=1}^{k} \frac{G_{j, a}(x)}{a(a+1)^{k-j+1}} .
\end{aligned}
$$

Since

$$
\begin{aligned}
\operatorname{Lif}_{-1}(z ; a) & =z \sum_{m=1}^{\infty} \frac{z^{m-1}}{(m-1) !}+a \sum_{m=0}^{\infty} \frac{z^{m}}{m !} \\
& =(z+a) e^{z},
\end{aligned}
$$

we get $G_{-1, a}(x)=(1+x)(a+\ln (1+x))$, so $a G_{0, a}(x)-G_{-1, a}(x)=-(1+x) \ln (1+x)$.

Hence,

$$
\begin{aligned}
& \sum_{\mu=1}^{k}\left(\frac{G_{\mu, a}(x)}{a^{k-\mu}}-\frac{G_{\mu-1, a}(x)}{a^{k-\mu+1}}\right) \\
& =\sum_{\mu=1}^{k} \frac{a G_{0, a}(x)-G_{-1, a}(x)}{a^{k-\mu+1}(a+1)^{\mu}} \\
& \quad+(1+x)^{2}(\ln (1+x))^{2}\left(\frac{d^{2}}{d x^{2}}+\frac{1}{1+x} \frac{d}{d x}\right) \sum_{\mu=1}^{k} \frac{1}{a^{k-\mu+1}} \sum_{j=1}^{\mu} \frac{G_{j, a}(x)}{(a+1)^{\mu-j+1}},
\end{aligned}
$$

entailing that

$$
\begin{aligned}
G_{k, a}(x)= & \frac{G_{0, a}(x)}{a^{k}}+\left(\frac{1}{a^{k}}-\frac{1}{(a+1)^{k}}\right)\left(a G_{0, a}(x)-G_{-1, a}(x)\right) \\
& +(1+x)^{2}(\ln (1+x))^{2}\left(\frac{d^{2}}{d x^{2}}+\frac{1}{1+x} \frac{d}{d x}\right) \sum_{j=1}^{k}(a+1)^{j} G_{j, a}(x) \sum_{\mu=j}^{k} \frac{1}{a^{k-\mu+1}(a+1)^{\mu+1}} \\
= & \frac{1+x}{a^{k}}-\left(\frac{1}{a^{k}}-\frac{1}{(a+1)^{k}}\right)(1+x) \ln (1+x) \\
& +(1+x)^{2}(\ln (1+x))^{2}\left(\frac{d^{2}}{d x^{2}}+\frac{1}{1+x} \frac{d}{d x}\right) \sum_{j=1}^{k}\left(\frac{1}{a^{k-j+1}}-\frac{1}{(a+1)^{k-j+1}}\right) G_{j, a}(x) .
\end{aligned}
$$

Therefore,

$$
\sum_{n=0}^{\infty} T_{3, a}^{(k)}(n) \frac{x^{n}}{n !}=\frac{x^{2}}{(\ln (1+x))^{2}} G_{k, a}(x)
$$




$$
\begin{aligned}
= & \frac{1}{a^{k}} \frac{x^{2}(1+x)}{(\ln (1+x))^{2}}-\left(\frac{1}{a^{k}}-\frac{1}{(a+1)^{k}}\right) \frac{x^{2}(1+x)}{\ln (1+x)} \\
& +x^{2}(1+x)^{2}\left(\frac{d^{2}}{d x^{2}}+\frac{1}{1+x} \frac{d}{d x}\right) \sum_{j=1}^{k}\left(\frac{1}{a^{k-j+1}}-\frac{1}{(a+1)^{k-j+1}}\right) G_{j, a}(x) \\
= & \sum_{n=0}^{\infty} \frac{T_{3, a}^{(0)}(n)}{a^{k}} \frac{x^{n}}{n !}-\left(\frac{1}{a^{k}}-\frac{1}{(a+1)^{k}}\right) x \sum_{n=0}^{\infty} T_{2, a}^{(0)}(n) \frac{x^{n}}{n !} \\
& +\sum_{j=1}^{k}\left(\frac{1}{a^{k-j+1}}-\frac{1}{(a+1)^{k-j+1}}\right) x^{2}(1+x)^{2}\left(\frac{d^{2}}{d x^{2}}+\frac{1}{1+x} \frac{d}{d x}\right) \sum_{n=0}^{\infty} c_{n, a}^{(j)} \frac{x^{n}}{n !} \\
= & \sum_{n=0}^{\infty} \frac{T_{3, a}^{(0)}(n)}{a^{k}} \frac{x^{n}}{n !}-\left(\frac{1}{a^{k}}-\frac{1}{(a+1)^{k}}\right) \sum_{n=0}^{\infty} n T_{2, a}^{(0)}(n-1) \frac{x^{n}}{n !} \\
& +\sum_{j=1}^{k}\left(\frac{1}{a^{k-j+1}}-\frac{1}{(a+1)^{k-j+1}}\right) \sum_{n=0}^{\infty} n(n-1)\left(c_{n, a}^{(j)}+(2 n-3) c_{n-1, a}^{(j)}+(n-2)^{2} c_{n-2, a}^{(j)}\right) \frac{x^{n}}{n !} .
\end{aligned}
$$

By comparing the coefficients of $x^{n} / n$ ! in both sides, we have the identity (17).

Finally, since

$$
\begin{aligned}
& (1+x)^{2}(\ln (1+x))^{2}\left(\frac{d^{2}}{d x^{2}}+\frac{1}{1+x} \frac{d}{d x}\right) \sum_{j=0}^{k-2}(a+1)^{k-j-2} G_{-j, a}(x) \\
& =\sum_{j=0}^{k-2}\left((a+1)^{k-j-1}\left(a G_{-j, a}(x)-G_{-j-1, a}(x)\right)-(a+1)^{k-j-2}\left(a G_{-j-1, a}(x)-G_{-j-2, a}(x)\right)\right) \\
& =(a+1)^{k-1}\left(a G_{0, a}(x)-G_{-1, a}(x)\right)-\left(a G_{-k+1, a}(x)-G_{-k, a}(x)\right),
\end{aligned}
$$

we obtain

$$
\begin{aligned}
& \sum_{\mu=2}^{k}\left(-a^{k-\mu+1} G_{-\mu+1, a}(x)+a^{k-\mu} G_{-\mu, a}(x)\right)=-a^{k-1} G_{-1, a}(x)+G_{-k, a}(x) \\
& =(1+x)^{2}(\ln (1+x))^{2}\left(\frac{d^{2}}{d x^{2}}+\frac{1}{1+x} \frac{d}{d x}\right) \sum_{\mu=2}^{k} \sum_{j=0}^{\mu-2} a^{k-\mu}(a+1)^{\mu-j-2} G_{-j, a}(x) \\
& \quad-\sum_{\mu=2}^{k} a^{k-\mu}(a+1)^{\mu-1}\left(a G_{0, a}(x)-G_{-1, a}(x)\right) \\
& =(1+x)^{2}(\ln (1+x))^{2}\left(\frac{d^{2}}{d x^{2}}+\frac{1}{1+x} \frac{d}{d x}\right) \sum_{j=0}^{k-2} a^{k}(a+1)^{-j-2} G_{-j, a}(x) \sum_{\mu=j+2}^{k} a^{-\mu}(a+1)^{\mu}
\end{aligned}
$$




$$
+\left((a+1)^{k}-a^{k-1}(a+1)\right)(1+x) \ln (1+x) .
$$

Hence, we have

$$
\begin{aligned}
G_{-k, a}(x)= & a^{k-1}(1+x)(a+\ln (1+x))+\left((a+1)^{k}-a^{k-1}(a+1)\right)(1+x) \ln (1+x) \\
& +(1+x)^{2}(\ln (1+x))^{2}\left(\frac{d^{2}}{d x^{2}}+\frac{1}{1+x} \frac{d}{d x}\right) \sum_{j=0}^{k-2}\left((a+1)^{k-j-1}-a^{k-j-1}\right) G_{-j, a}(x) \\
= & a^{k}(1+x)+\left((a+1)^{k}-a^{k}\right)(1+x) \ln (1+x) \\
& +(1+x)^{2}(\ln (1+x))^{2}\left(\frac{d^{2}}{d x^{2}}+\frac{1}{1+x} \frac{d}{d x}\right) \sum_{j=0}^{k-2}\left((a+1)^{k-j-1}-a^{k-j-1}\right) G_{-j, a}(x) .
\end{aligned}
$$

Therefore,

$$
\begin{aligned}
& \sum_{n=0}^{\infty} T_{3, a}^{(-k)}(n) \frac{x^{n}}{n !}=\frac{x^{2}}{(\ln (1+x))^{2}} G_{-k, a}(x) \\
& =a^{k} \frac{x^{2}(1+x)}{(\ln (1+x))^{2}}+\left((a+1)^{k}-a^{k}\right) \frac{x^{2}(1+x)}{\ln (1+x)} \\
& \quad+x^{2}(1+x)^{2}\left(\frac{d^{2}}{d x^{2}}+\frac{1}{1+x} \frac{d}{d x}\right) \sum_{j=0}^{k-2}\left((a+1)^{k-j-1}-a^{k-j-1}\right) G_{-j, a}(x) \\
& =\sum_{n=0}^{\infty} a^{k} T_{3, a}^{(0)}(n) \frac{x^{n}}{n !}+\left((a+1)^{k}-a^{k}\right) \sum_{n=0}^{\infty} n T_{2, a}^{(0)}(n-1) \frac{x^{n}}{n !} \\
& \quad+\sum_{j=0}^{k-2}\left((a+1)^{k-j-1}-a^{k-j-1}\right) \sum_{n=0}^{\infty} n(n-1)\left(c_{n, a}^{(-j)}+(2 n-3) c_{n-1, a}^{(-j)}+(n-2)^{2} c_{n-2, a}^{(-j)}\right) \frac{x^{n}}{n !},
\end{aligned}
$$

yielding the identity (18).

2.3. Explicit formulae for $T_{4, a}^{(k)}(n)$. Kamano [14] mentioned that explicit formulae of $S_{m}^{(k)}$ for $m \geq 4$ seemed to be complicated to describe. However, similarly to Theorem 2 and Theorem 3, we can obtain explicit forms for $T_{4, a}^{(k)}(n)$.

THEOREM 4. For integers $n \geq 0$ and $k \geq 1$ and a real number $a>0$, we have

$$
\begin{aligned}
T_{4, a}^{(0)}(n)= & T_{3,1}^{(1)}(n)+n T_{3,1}^{(1)}(n-1), \\
T_{4, a}^{(k)}(n)= & \frac{T_{4, a}^{(0)}(n)}{a^{k}}-n\left(\frac{1}{a^{k}}-\frac{1}{(a+1)^{k}}\right) T_{3, a}^{(0)}(n-1) \\
& +n(n-1)\left(\frac{1}{2 a^{k}}-\frac{1}{(a+1)^{k}}+\frac{1}{2(a+2)^{k}}\right) T_{2,1}^{(0)}(n-2)
\end{aligned}
$$




$$
\begin{aligned}
- & n(n-1)(n-2) \sum_{j=1}^{k}\left(\frac{1}{2 a^{k-j+1}}-\frac{1}{(a+1)^{k-j+1}}+\frac{1}{2(a+2)^{k-j+1}}\right) \\
& \times\left(c_{n, a}^{(j)}+(3 n-6) c_{n-1, a}^{(j)}+\left(3 n^{2}-15 n+19\right) c_{n-2, a}^{(j)}+(n-3)^{3} c_{n-3, a}^{(j)}\right),
\end{aligned}
$$

$$
\begin{aligned}
T_{4, a}^{(-k)}(n)= & a^{k} T_{4, a}^{(0)}(n)+n\left((a+1)^{k}-a^{k}\right) T_{3, a}^{(0)}(n-1) \\
& +n(n-1)\left(\frac{(a+2)^{k}}{2}-(a+1)^{k}+\frac{a^{k}}{2}\right) T_{2, a}^{(0)}(n-2) \\
& +n(n-1)(n-2) \sum_{j=0}^{k-3}\left(\frac{(a+2)^{k-j-1}}{2}-(a+1)^{k-j-1}+\frac{a^{k-j-1}}{2}\right) \\
& \times\left(c_{n, a}^{(-j)}+(3 n-6) c_{n-1, a}^{(-j)}+\left(3 n^{2}-15 n+19\right) c_{n-2, a}^{(-j)}+(n-3)^{3} c_{n-3, a}^{(-j)}\right) .
\end{aligned}
$$

2.4. Explicit formulae for $T_{m, a}^{(k)}(n)$. Continually, we can also obtain explicit forms of $T_{m, a}^{(k)}(n)$ for $m=5,6, \ldots$ Actually, there exist explicit formulae of $T_{m, a}^{(k)}(n)$ for any general integer $m \geq 2$.

THEOREM 5. For integers $n \geq 0$ and $k \geq 1$ and a real number $a>0$, we have

$$
\begin{aligned}
T_{m, a}^{(0)}(n)= & T_{m-1,1}^{(1)}(n)+n T_{m-1,1}^{(1)}(n-1) \\
T_{m, a}^{(k)}(n)= & \sum_{r=0}^{m-2}\left(\begin{array}{l}
n \\
r
\end{array}\right) \sum_{i=0}^{r}\left(\begin{array}{l}
r \\
i
\end{array}\right) \frac{(-1)^{r-i} T_{m-r, a}^{(0)}(n-r)}{(a+i)^{k}} \\
& +n\left(\begin{array}{c}
n-1 \\
m-2
\end{array}\right) \sum_{j=1}^{k} \sum_{i=0}^{m-2}\left(\begin{array}{c}
m-2 \\
i
\end{array}\right) \frac{(-1)^{m-i-1}}{(a+i)^{k-j+1}} \sum_{\kappa=0}^{m-1} P_{m, \kappa}(n) c_{n-\kappa, a}^{(j)}, \\
T_{m, a}^{(-k)}(n)= & \sum_{r=0}^{m-2}\left(\begin{array}{c}
n \\
r
\end{array}\right) \sum_{i=0}^{r}\left(\begin{array}{c}
r \\
i
\end{array}\right)(-1)^{r-i}(a+i)^{k} T_{m-r, a}^{(0)}(n-r) \\
& +n\left(\begin{array}{c}
n-1 \\
m-2
\end{array}\right) \sum_{j=0}^{k-m+1} \sum_{i=0}^{m-2}\left(\begin{array}{c}
m-2 \\
i
\end{array}\right)(-1)^{m-i}(a+i)^{k-j-1} \sum_{\kappa=0}^{m-1} P_{m, \kappa}(n) c_{n-\kappa, a}^{(-j)},
\end{aligned}
$$

where

$$
P_{m, \kappa}(n)=\sum_{t=0}^{\kappa}\left\{\begin{array}{c}
m-1 \\
m-t-1
\end{array}\right\}\left(\begin{array}{l}
m-t-1 \\
m-\kappa-1
\end{array}\right) \frac{(n-m+1) !}{(n-m-\kappa+t+1) !} \quad(\kappa=0,1, \ldots, m-2)
$$


and

$$
P_{m, m-1}(n)=\sum_{t=0}^{m-2}\left\{\begin{array}{c}
m-1 \\
m-t-1
\end{array}\right\} \frac{(n-m+1) !}{(n-2 m+t+2) !}=(n-m+1)^{m-1} .
$$

\subsection{Proof of Theorem 5. Since}

$$
\begin{aligned}
\sum_{n=0}^{\infty} T_{m, a}^{(0)}(n) \frac{x^{n}}{n !} & =\left(\frac{x}{\ln (1+x)}\right)^{m-1} \operatorname{Lif}_{0}(\ln (1+x) ; a) \\
& =\left(\frac{x}{\ln (1+x)}\right)^{m-1}(1+x) \\
& =\left(\frac{x}{\ln (1+x)}\right)^{m-1}+x\left(\frac{x}{\ln (1+x)}\right)^{m-1} \\
& =\sum_{n=0}^{\infty} T_{m-1,1}^{(1)}(n) \frac{x^{n}}{n !}+\sum_{n=0}^{\infty}(n+1) T_{m-1,1}^{(1)}(n) \frac{x^{n+1}}{(n+1) !}
\end{aligned}
$$

the identity (22) holds.

We shall prove the identity (23) in a different method from those used in the proofs in Theorem 2 and Theorem 3. The identity (24) can be proven similarly and omitted. We need some lemmata.

LEMMA 2. For any integer $m \geq 1$ we have

$$
\begin{aligned}
& \frac{1}{n+a-y}=\sum_{r=0}^{m-1}(-1)^{r}\left(\begin{array}{l}
n \\
r
\end{array}\right) \sum_{i=0}^{r}\left(\begin{array}{l}
r \\
i
\end{array}\right) \frac{(-1)^{i}}{i+a-y} \\
& +\sum_{i=0}^{m-1} \frac{(-1)^{m+i} m !\left(\begin{array}{l}
n \\
m
\end{array}\right)}{i !(m-i-1) !(n-i)}\left(\frac{1}{i+a-y}-\frac{1}{n+a-y}\right) .
\end{aligned}
$$

ProOF. Put

$$
\begin{aligned}
h_{n}(m) & =\sum_{r=0}^{m-1}(-1)^{r}\left(\begin{array}{l}
n \\
r
\end{array}\right) \sum_{i=0}^{r}\left(\begin{array}{l}
r \\
i
\end{array}\right) \frac{(-1)^{i}}{i+a-y} \\
& +\sum_{i=0}^{m-1} \frac{(-1)^{m+i} m !\left(\begin{array}{l}
n \\
m
\end{array}\right)}{i !(m-i-1) !(n-i)}\left(\frac{1}{i+a-y}-\frac{1}{n+a-y}\right) .
\end{aligned}
$$

For $m=1$

$$
h_{n}(1)=\frac{1}{a-y}-\left(\frac{1}{a-y}-\frac{1}{n+a-y}\right)=\frac{1}{n+a-y} .
$$


For $m \geq 1$

$$
\begin{aligned}
& h_{n}(m+1)-h_{n}(m) \\
&=(-1)^{m}\left(\begin{array}{c}
n \\
m
\end{array}\right) \sum_{i=0}^{m}\left(\begin{array}{c}
m \\
i
\end{array}\right) \frac{(-1)^{i}}{i+a-y} \\
&+\sum_{i=0}^{m} \frac{(-1)^{m+i+1}(m+1) !\left(\begin{array}{c}
n \\
m+1
\end{array}\right)}{i !(m-i) !(n-i)}\left(\frac{1}{i+a-y}-\frac{1}{n+a-y}\right) \\
& \quad-\sum_{i=0}^{m-1} \frac{(-1)^{m+i} m !\left(\begin{array}{c}
n \\
m
\end{array}\right)}{i !(m-i-1) !(n-i)}\left(\frac{1}{i+a-y}-\frac{1}{n+a-y}\right) \\
&=(-1)^{m}\left(\begin{array}{c}
n \\
m
\end{array}\right) \sum_{i=0}^{m}\left(\begin{array}{c}
m \\
i
\end{array}\right) \frac{(-1)^{i}}{i+a-y}-\left(\begin{array}{c}
n \\
m
\end{array}\right)\left(\frac{1}{m+a-y}-\frac{1}{n+a-y}\right) \\
&+\sum_{i=0}^{m-1}(-1)^{m+i+1}\left(\begin{array}{c}
n \\
m
\end{array}\right)\left(\begin{array}{c}
m \\
i
\end{array}\right)\left(\frac{1}{i+a-y}-\frac{1}{n+a-y}\right) \\
&=\left(\begin{array}{l}
n \\
m
\end{array}\right) \frac{1}{m+a-y}-\left(\begin{array}{c}
n \\
m
\end{array}\right)\left(\frac{1}{m+a-y}-\frac{1}{n+a-y}\right) \\
&+\frac{(-1)^{m}}{n+a-y}\left(\begin{array}{c}
n \\
m
\end{array}\right) \sum_{i=0}^{m-1}\left(\begin{array}{c}
m \\
i
\end{array}\right)(-1)^{i} \\
&=\left(\begin{array}{l}
n \\
m
\end{array}\right) \frac{1}{n+a-y}-\left(\begin{array}{l}
n \\
m
\end{array}\right) \frac{1}{n+a-y}=0 .
\end{aligned}
$$

LEMMA 3. For $m \geq 1$ and $n \geq 0$ we have

$$
\begin{aligned}
& \frac{1}{(n+a)^{k}} \\
& \quad=\sum_{r=0}^{m-1}(-1)^{r}\left(\begin{array}{l}
n \\
r
\end{array}\right) \sum_{i=0}^{r}\left(\begin{array}{l}
r \\
i
\end{array}\right) \frac{(-1)^{i}}{(a+i)^{k}}+\sum_{j=1}^{k} \sum_{i=0}^{m-1} \frac{(-1)^{m+i}\left(\begin{array}{c}
n \\
m
\end{array}\right) m !}{i !(m-i-1) !(i+a)^{k-j+1}(n+a)^{j}} .
\end{aligned}
$$

PROOF. Taking the infinite sum $\sum_{k=1}^{\infty} y^{k-1}$ for small $y>0$, the left-hand side of the identity in Lemma 3 is equal to

$$
\sum_{k=1}^{\infty} \frac{y^{k-1}}{(n+a)^{k}}=\frac{1}{n+a-y} .
$$


By Lemma 2, the right-hand side is equal to

$$
\begin{aligned}
& \sum_{r=0}^{m-1}(-1)^{r}\left(\begin{array}{l}
n \\
r
\end{array}\right) \sum_{i=0}^{r}\left(\begin{array}{l}
r \\
i
\end{array}\right) \frac{(-1)^{i}}{i+a} \sum_{k=1}^{\infty}\left(\frac{y}{i+a}\right)^{k-1} \\
& \quad+\sum_{i=0}^{m-1} \frac{(-1)^{m+i} m !\left(\begin{array}{l}
n \\
m
\end{array}\right)}{i !(m-i-1) !} \sum_{k=1}^{\infty} \frac{y^{k-1}}{(i+a)^{k}} \sum_{j=1}^{k} \frac{(i+a)^{j-1}}{(n+a)^{j}} \\
& =\sum_{r=0}^{m-1}(-1)^{r}\left(\begin{array}{l}
n \\
r
\end{array}\right) \sum_{i=0}^{r}\left(\begin{array}{l}
r \\
i
\end{array}\right) \frac{(-1)^{i}}{i+a-y} \\
& \quad+\sum_{i=0}^{m-1} \frac{(-1)^{m+i} m !\left(\begin{array}{l}
n \\
m
\end{array}\right)}{i !(m-i-1) !} \frac{1}{n-i}\left(\frac{1}{i+a-y}-\frac{1}{n+a-y}\right) \\
& =\frac{1}{n+a-y} .
\end{aligned}
$$

LEMMA 4. For $m \geq 0$ we have

$$
\sum_{l=0}^{m}(-1)^{l} f_{m, l}(n+a)^{l}=(-1)^{m} m !\left(\begin{array}{l}
n \\
m
\end{array}\right) .
$$

Proof. By the definition of $f_{m, l}$ in (9)

$$
\begin{aligned}
\sum_{l=0}^{m}(-1)^{l} f_{m, l}(n+a)^{l} & =(-1)^{m}((n+a)-a)((n+a)-(a+1)) \cdots((n+a)-(a+m-1)) \\
& =(-1)^{m} m !\left(\begin{array}{l}
n \\
m
\end{array}\right) .
\end{aligned}
$$

By taking the summation $\sum_{n=0}^{\infty}(\ln (1+x))^{n} / n$ ! on both sides of the identity in Lemma 3 and by Lemma 4, we have

$$
\begin{aligned}
G_{k, a}(x)= & \sum_{n=0}^{\infty} \frac{(\ln (1+x))^{n}}{n !(n+a)^{k}} \\
= & \sum_{r=0}^{m-1}(-1)^{r} \sum_{i=0}^{r}\left(\begin{array}{l}
r \\
i
\end{array}\right) \frac{(-1)^{i}}{(i+a)^{k}} \frac{(\ln (1+x))^{r}}{r !} \sum_{n=r}^{\infty} \frac{(\ln (1+x))^{n-r}}{(n-r) !} \\
& +\sum_{j=1}^{k} \sum_{i=0}^{m-1} \frac{(-1)^{m+i} m !}{i !(m-i-1) !(i+a)^{k-j+1}} \sum_{n=0}^{\infty}\left(\begin{array}{l}
n \\
m
\end{array}\right) \frac{(\ln (1+x))^{n}}{n !(n+a)^{j}}
\end{aligned}
$$




$$
\begin{aligned}
= & \sum_{r=0}^{m-1} \frac{(1+x)(-\ln (1+x))^{r}}{r !} \sum_{i=0}^{r}\left(\begin{array}{l}
r \\
i
\end{array}\right) \frac{(-1)^{i}}{(i+a)^{k}} \\
& +\sum_{j=1}^{k} \sum_{i=0}^{m-1} \frac{(-1)^{m-i}}{i !(m-i-1) !(i+a)^{k-j+1}} \sum_{l=0}^{m}(-1)^{m-l} f_{m, l} G_{j-l, a}(x) .
\end{aligned}
$$

Replacing $m$ by $m-1$ and $k$ by $j$ in Lemma 1 , and multiplying both sides by $(1+$ $x)^{m-1} x^{m-1}$, we have

$$
\begin{aligned}
& \left.\frac{x}{\ln (1+x)}\right)^{m-1} \sum_{l=0}^{m-1}(-1)^{m-l-1} f_{m-1, l} G_{j-l, a}(x) \\
= & (1+x)^{m-1} x^{m-1} \sum_{t=0}^{m-2}\left\{\begin{array}{c}
m-1 \\
m-t-1
\end{array}\right\} \frac{1}{(1+x)^{t}} \frac{d^{m-t-1}}{d x^{m-t-1}} G_{j, a}(x) \\
= & (1+x)^{m-1} x^{m-1} \sum_{t=0}^{m-2}\left\{\begin{array}{c}
m-1 \\
m-t-1
\end{array}\right\} \frac{1}{(1+x)^{t}} \sum_{n=0}^{\infty} c_{m+n-t-1, a}^{(j)} \frac{x^{n}}{n !} \\
= & \sum_{t=0}^{m-2}\left\{\begin{array}{c}
m-1 \\
m-t-1
\end{array}\right\}(1+x)^{m-t-1} \sum_{n=0}^{\infty} \frac{n !}{(n-m+1) !} c_{n-t, a}^{(j)} \frac{x^{n}}{n !} \\
= & \sum_{t=0}^{m-2}\left\{\begin{array}{c}
m-1 \\
m-t-1
\end{array}\right\} \sum_{r=0}^{m-t-1}\left(\begin{array}{c}
m-t-1 \\
r
\end{array}\right) x^{r} \sum_{n=0}^{\infty} \frac{n !}{(n-m+1) !} c_{n-t, a}^{(j)} \frac{x^{n}}{n !} \\
= & \sum_{t=0}^{m-2}\left\{\begin{array}{c}
m-1 \\
m-t-1
\end{array}\right\} \sum_{r=0}^{m-t-1}\left(\begin{array}{c}
m-t-1 \\
r
\end{array}\right) \sum_{n=0}^{\infty} \frac{n !}{(n-r-m+1) !} c_{n-r-t, a}^{(j)} \frac{x^{n}}{n !} \\
= & \sum_{t=0}^{m-2}\left\{\begin{array}{c}
m-1 \\
m-t-1
\end{array}\right\} \sum_{\kappa=0}^{m-1}\left(\begin{array}{c}
m-t-1 \\
m-\kappa-1
\end{array}\right) \sum_{n=0}^{\infty} \frac{n !}{(n-\kappa+t-m+1) !} c_{n-\kappa, a}^{(j)} \frac{x^{n}}{n !} \quad(\kappa=r+t) \\
= & \sum_{n=0}^{\infty} \frac{n !}{(n-m+1) !} \sum_{\kappa=0}^{m-1} P_{m, \kappa}(n) c_{n-\kappa, a}^{(j)} \frac{x^{n}}{n !} \cdot \quad
\end{aligned}
$$

Thus, replacing $m$ by $m-1$ and multiplying $(x / \ln (1+x))^{m-1}$ on both sides of (25),

$$
\begin{aligned}
& \left(\frac{x}{\ln (1+x)}\right)^{m-1} G_{n, a}^{(k)}(x)=\sum_{n=0}^{\infty} T_{m, a}^{(k)}(n) \frac{x^{n}}{n !} \\
& =\sum_{r=0}^{m-2} \frac{(-x)^{r}}{r !} \sum_{i=0}^{r}\left(\begin{array}{l}
r \\
i
\end{array}\right) \frac{(-1)^{i}}{(i+a)^{k}} \sum_{n=r}^{\infty} T_{m-r, a}^{(0)}(n-r) \frac{x^{n-r}}{(n-r) !}
\end{aligned}
$$




$$
+\sum_{n=0}^{\infty} \frac{n !}{(n-m+1) !} \sum_{j=1}^{k} \sum_{i=0}^{m-2} \frac{(-1)^{m-i-1}}{i !(m-i-2) !(i+a)^{k-j+1}} \sum_{\kappa=0}^{m-1} P_{m, \kappa}(n) c_{n-\kappa, a}^{(j)} \frac{x^{n}}{n !} .
$$

Comparing the coefficients, we obtain the identity (23).

\section{Poly-Cauchy numbers of the second kind}

Similarly to $T_{m, a}^{(k)}$, define $\hat{T}_{m, a}^{(k)}(n)$ for a real number $a>0$ by

$$
\hat{T}_{m, a}^{(k)}(n):=\sum_{\substack{i_{1}+\ldots+i_{m}=n \\
i_{1}, \ldots, i_{m} \geq 0}}\left(\begin{array}{c}
n \\
i_{1}, \ldots, i_{m}
\end{array}\right) \underbrace{\hat{c}_{i_{1}} \cdots \hat{c}_{i_{m-1}}}_{m-1} \hat{c}_{i_{m}, a}^{(k)} \quad(m \geq 1, n \geq 0),
$$

where $\hat{c}_{n, a}^{(k)}$ is a generalized poly-Cauchy number of the second kind, whose generating function is given by

$$
\operatorname{Lif}_{k}(-\ln (1+x) ; a)=\sum_{n=0}^{\infty} \hat{c}_{n, a}^{(k)} \frac{x^{n}}{n !}
$$

$\hat{c}_{n, a}^{(k)}$ can be also defined by

$$
\hat{c}_{n, a}^{(k)}=(-1)^{a} \underbrace{\int_{0}^{1} \cdots \int_{0}^{1}}_{k}\left(-x_{1} x_{2} \cdots x_{k}\right)^{a}\left(-x_{1} x_{2} \cdots x_{k}-1\right) \cdots\left(-x_{1} x_{2} \cdots x_{k}-n+1\right) d x_{1} d x_{2} \cdots d x_{k} .
$$

When $a=k=1, \hat{c}_{n, 1}^{(1)}=\hat{c}_{n}$ is the classical Cauchy number of the second kind, whose generating function is given by

$$
\frac{x}{(1+x) \ln (1+x)}=\sum_{n=0}^{\infty} \hat{c}_{n} \frac{x^{n}}{n !} .
$$

By using the corresponding lemma to Lemma 1 , where $G_{k, a}(x)$ is replaced by $\hat{G}_{k, a}(x)=$ $\operatorname{Lif}_{k}(-\ln (1+x) ; a)$, we can obtain the following result.

THEOREM 6. For an integer $k$ and a non-negative integer $m$, we have

$$
\begin{aligned}
& \sum_{l=0}^{m}(-1)^{m-l} f_{m, l} \hat{T}_{m+1, a}^{(k-l)}(n) \\
= & \begin{cases}\sum_{l=0}^{m-1} \sum_{i=0}^{n-m}(-1)^{n-m-i} \frac{n !}{i !}\left(\begin{array}{c}
n-l-i-1 \\
n-m-i
\end{array}\right)\left\{\begin{array}{c}
m \\
l
\end{array}\right\} \hat{c}_{l+i, a}^{(k)}+\frac{n !}{(n-m) !} \hat{c}_{n, a}^{(k)} & (n \geq m) ; \\
0 & (0 \leq n \leq m-1) .\end{cases}
\end{aligned}
$$


Proof.

$$
\begin{aligned}
& \sum_{n=0}^{\infty} \sum_{l=0}^{m}(-1)^{m-l} f_{m, l} \hat{T}_{m+1, a}^{(k-l)}(n) \frac{x^{n}}{n !} \sum_{l=0}^{m}(-1)^{m-l} f_{m, l}\left(\frac{x}{(1+x) \ln (1+x)}\right)^{m} \hat{G}_{k-l, a}(x) \\
&= x^{m} \sum_{l=0}^{m} \frac{1}{(1+x)^{m-l}}\left\{\begin{array}{c}
m \\
l
\end{array}\right\} \sum_{i=0}^{\infty} \hat{c}_{l+i, a}^{(k)} \frac{x^{i}}{i !} \\
&= x^{m} \sum_{l=0}^{m}\left(\sum_{\nu=0}^{\infty}(-1)^{\nu}\left(\begin{array}{c}
m-l+v-1 \\
v
\end{array}\right) x^{\nu}\right)\left\{\begin{array}{c}
m \\
l
\end{array}\right\} \sum_{i=0}^{\infty} \frac{\hat{c}_{l+i, a}^{(k)}}{i !} x^{i} \\
&= \sum_{n=0}^{\infty} \sum_{l=0}^{m} \sum_{i=0}^{n}(-1)^{n-i}\left(\begin{array}{c}
m-l+n-i-1 \\
n-i
\end{array}\right)\left\{\begin{array}{c}
m \\
l
\end{array}\right\} \frac{(n+m) !}{i !} \hat{c}_{l+i, a}^{(k)} \frac{x^{n+m}}{(n+m) !} \\
&= \sum_{n=m}^{\infty}\left(\sum_{l=0}^{m-1} \sum_{i=0}^{n-m}(-1)^{n-m-i} \frac{n !}{i !}\left(\begin{array}{c}
n-l-i-1 \\
n-m-i
\end{array}\right)\left\{\begin{array}{c}
m \\
l
\end{array}\right\} \hat{c}_{l+i, a}^{(k)}+\frac{n !}{(n-m) !} \hat{c}_{n, a}^{(k)}\right) \\
& n
\end{aligned}
$$

Putting $m=1$ in Theorem 6, we have the following. This is also an analogous formula to Euler's identity (3).

COROLlary 3.

$$
\sum_{i=0}^{n}\left(\begin{array}{l}
n \\
i
\end{array}\right) \hat{c}_{i}\left(\hat{c}_{n-i, a}^{(k-1)}-a \hat{c}_{n-i, a}^{(k)}\right)=n \hat{c}_{n, a}^{(k)} \quad(n \geq 0) .
$$

3.1. Explicit formulae for $\hat{T}_{m, a}^{(k)}(n)$. Similarly to Theorem 5 , the following result holds for a general $m \geq 2$.

THEOREM 7. For integers $n \geq 0$ and $k \geq 1$ and a real number $a>0$ we have

$$
\begin{aligned}
& \hat{T}_{m, a}^{(0)}(n)=\sum_{l=0}^{n-l}(-1)^{n-l} \frac{n !}{l !} \hat{T}_{m-1,1}^{(1)}(l) \\
& \hat{T}_{m, a}^{(k)}(n)=\frac{\hat{T}_{m, a}^{(0)}(n)}{a^{k}}+\sum_{r=1}^{m-2} \frac{1}{r !} \sum_{i=0}^{r}\left(\begin{array}{l}
r \\
i
\end{array}\right) \frac{(-1)^{i+n-r}}{(i+a)^{k}} \sum_{l=0}^{n-r}\left(\begin{array}{c}
n-l-1 \\
r-1
\end{array}\right)(-1)^{l} \frac{n !}{l !} \hat{T}_{m-r, a}^{(0)}(l) \\
& +n ! \sum_{j=1}^{k} \sum_{i=0}^{m-2} \frac{(-1)^{m-i-1}}{i !(m-i-2) !(i+a)^{k-j+1}}
\end{aligned}
$$




$$
\begin{aligned}
& \quad \times\left(\frac{\hat{c}_{n, a}^{(j)}}{(n-m+1) !}+\sum_{t=1}^{m-2}\left\{\begin{array}{c}
m-1 \\
m-t-1
\end{array}\right\} \sum_{l=0}^{n-m+1}(-1)^{n-m-l+1}\left(\begin{array}{c}
n+t-m-l \\
t-1
\end{array}\right) \frac{\hat{c}_{m+l-t-1, a}^{(j)}}{l !}\right), \\
& \hat{T}_{m, a}^{(-k)}(n)=a^{k} \hat{T}_{m, a}^{(0)}(n)+\sum_{r=1}^{m-2} \frac{1}{r !} \sum_{i=0}^{r}\left(\begin{array}{l}
r \\
i
\end{array}\right)(-1)^{i+n-r}(i+a)^{k} \sum_{l=0}^{n-r}\left(\begin{array}{c}
n-l-1 \\
r-1
\end{array}\right)(-1)^{l} \frac{n !}{l !} \hat{T}_{m-r, a}^{(0)}(l) \\
& +n ! \sum_{j=0}^{k-m+1} \sum_{i=0}^{m-2} \frac{(-1)^{m-i}(i+a)^{k-j-1}}{i !(m-i-2) !} \\
& \quad \times\left(\frac{\hat{c}_{n, a}^{(-j)}}{(n-m+1) !}+\sum_{t=1}^{m-2}\left\{\begin{array}{c}
m-1 \\
m-t-1
\end{array}\right\} \sum_{l=0}^{n-m+1}(-1)^{n-m-l+1}\left(\begin{array}{c}
n+t-m-l \\
t-1
\end{array}\right) \frac{\hat{c}_{m+l-t-1, a}^{(-j)}}{l !}\right) .
\end{aligned}
$$

3.2. Proof of Theorem 7. Firstly,

$$
\begin{aligned}
\sum_{n=0}^{\infty} \hat{T}_{m, a}^{(0)}(n) \frac{x^{n}}{n !} & =\left(\frac{x}{(1+x) \ln (1+x)}\right)^{m-1} \hat{G}_{0, a}(x) \\
& =\frac{1}{1+x}\left(\frac{x}{(1+x) \ln (1+x)}\right)^{m-1} \\
& =\frac{1}{1+x}\left(\frac{x}{(1+x) \ln (1+x)}\right)^{m-2} \hat{G}_{1,1}(x) \\
& =\frac{1}{1+x} \sum_{n=0}^{\infty} \hat{T}_{m-2,1}^{(1)}(n) \frac{x^{n}}{n !} \\
& =\sum_{\rho=0}^{\infty}(-1)^{\rho} x^{\rho} \sum_{\nu=0}^{\infty} \frac{\hat{T}_{m-2,1}^{(1)}(v)}{\nu !} x^{\nu} \\
& =\sum_{n=0}^{\infty}\left(\sum_{l=0}^{n}(-1)^{n-l} \frac{\hat{T}_{m-2,1}^{(1)}(l)}{l !}\right) x^{n} \\
& =\sum_{n=0}^{\infty}\left(\sum_{l=0}^{n}(-1)^{n-l} \frac{n !}{l !} \hat{T}_{m-2,1}^{(1)}(l)\right) \frac{x^{n}}{n !}
\end{aligned}
$$

Hence, the identity (26) holds.

Notice that

$$
\begin{aligned}
\frac{x^{m-1}}{(1+x)^{m}(\ln (1+x))^{m-r-1}} & =\left(\frac{x}{1+x}\right)^{r}\left(\frac{x}{(1+x) \ln (1+x)}\right)^{m-r-1} \frac{1}{1+x} \\
& =\left(\frac{x}{1+x}\right)^{r} \sum_{n=0}^{\infty} T_{m-r, a}^{(0)}(n) \frac{x^{n}}{n !}
\end{aligned}
$$




$$
\begin{aligned}
& =x^{r}\left(\sum_{\mu=0}^{\infty}(-1)^{\mu}\left(\begin{array}{c}
\mu+r-1 \\
\mu
\end{array}\right) x^{\mu}\right)\left(\sum_{l=0}^{\infty} T_{m-r, a}^{(0)}(l) \frac{x^{l}}{l !}\right) \\
& =x^{r} \sum_{n=0}^{\infty} \sum_{l=0}^{n}(-1)^{n-l}\left(\begin{array}{c}
n+r-l-1 \\
r-1
\end{array}\right) \frac{T_{m-r, a}^{(0)}(l)}{l !} x^{n} \\
& =\sum_{n=0}^{\infty} \sum_{l=0}^{n}(-1)^{n-l}\left(\begin{array}{c}
n+r-l-1 \\
r-1
\end{array}\right)(n+r) ! \frac{T_{m-r, a}^{(0)}(l)}{l !} \frac{x^{n+r}}{(n+r) !} \\
& =\sum_{n=0}^{\infty} \sum_{l=0}^{n-r}(-1)^{n-r-l}\left(\begin{array}{c}
n-l-1 \\
r-1
\end{array}\right) \frac{n !}{l !} T_{m-r, a}^{(0)}(l) \frac{x^{n}}{n !} .
\end{aligned}
$$

Hence, similarly to the identity (25), by replacing $m$ by $m-1$, for $k \geq 1$ and $m \geq 2$ we have

$$
\begin{aligned}
\hat{G}_{k, a}(x) & =\frac{\hat{G}_{0, a}(x)}{a^{k}}+\sum_{r=1}^{m-2} \frac{(\ln (1+x))^{r}}{(1+x) r !} \sum_{i=0}^{r}\left(\begin{array}{l}
r \\
i
\end{array}\right) \frac{(-1)^{i}}{(i+a)^{k}} \\
+\sum_{j=1}^{k} & \sum_{i=0}^{m-2} \frac{(-1)^{m-i-1}}{i !(m-i-2) !(i+a)^{k-j+1}} \sum_{l=0}^{m-1}(-1)^{l} f_{m-1, l} \hat{G}_{j-l, a}(x) .
\end{aligned}
$$

Now, we have

$$
\begin{aligned}
& \left(\frac{x}{(1+x) \ln (1+x)}\right)^{m-1} \sum_{l=0}^{m-1}(-1)^{m-l-1} f_{m-1, l} \hat{G}_{j-l, a}(x) \\
= & x^{m-1} \sum_{t=0}^{m-2}\left\{\begin{array}{c}
m-1 \\
m-t-1
\end{array}\right\} \frac{1}{(1+x)^{t}} \frac{d^{m-t-1}}{d x^{m-t-1}} \hat{G}_{j, a}(x) \\
= & x^{m-1} \sum_{t=0}^{m-2}\left\{\begin{array}{c}
m-1 \\
m-t-1
\end{array}\right\} \frac{1}{(1+x)^{t}} \sum_{n=0}^{\infty} \hat{c}_{m+n-t-1, a}^{(j)} \frac{x^{n}}{n !} \\
= & \sum_{t=0}^{m-2}\left\{\begin{array}{c}
m-1 \\
m-t-1
\end{array}\right\} \frac{1}{(1+x)^{t}} \sum_{n=0}^{\infty} \frac{n !}{(n-m+1) !} \hat{c}_{n-t, a}^{(j)} \frac{x^{n}}{n !} \\
= & \sum_{n=0}^{\infty} \frac{n !}{(n-m+1) !} \hat{c}_{n, a}^{(j)} \frac{x^{n}}{n !} \\
& +\sum_{t=1}^{m-2}\left\{\begin{array}{c}
m-1 \\
m-t-1
\end{array}\right\}\left(\sum_{\mu=0}^{\infty}(-1)^{\mu}\left(\begin{array}{c}
t+\mu-1 \\
\mu
\end{array}\right) x^{\mu}\right)\left(\sum_{\nu=0}^{\infty} \frac{\nu !}{(\nu-m+1) !} \hat{c}_{\nu-t, a}^{(j)} \frac{x^{\nu}}{\nu !}\right) \\
= & \sum_{n=0}^{\infty} \frac{n !}{(n-m+1) !} \hat{c}_{n, a}^{(j)} \frac{x^{n}}{n !}
\end{aligned}
$$




$$
\begin{aligned}
& +\sum_{t=1}^{m-2}\left\{\begin{array}{c}
m-1 \\
m-t-1
\end{array}\right\} \sum_{n=0}^{\infty} \sum_{\nu=0}^{n}(-1)^{n-v}\left(\begin{array}{c}
t+n-v-1 \\
t-1
\end{array}\right) \frac{n !}{(v-m+1) !} \hat{c}_{v-t, a}^{(j)} \frac{x^{n}}{n !} \\
= & \sum_{n=0}^{\infty} n !\left(\frac{\hat{c}_{n, a}^{(j)}}{(n-m+1) !}\right. \\
& \left.+\sum_{t=1}^{m-2}\left\{\begin{array}{c}
m-1 \\
m-t-1
\end{array}\right\} \sum_{l=0}^{n-m+1}(-1)^{n-m-l+1}\left(\begin{array}{c}
n+t-m-l \\
t-1
\end{array}\right) \frac{\hat{c}_{m+l-t-1, a}^{(j)}}{l !}\right) \frac{x^{n}}{n !} .
\end{aligned}
$$

By multiplying $(x /(1+x) \ln (1+x))^{m-1}$ on both sides of (29) and comparing the coefficients of $x^{n} / n$ !, we obtain the identity (27). The identity (28) is similarly proven.

3.3. Explicit formulae of $\hat{T}_{2, a}^{(k)}(n)$ and $\hat{T}_{3, a}^{(k)}(n)$. Putting $m=2$ in Theorem 7, we have the following identities. This is an extension of [20, Theorem 9].

THEOREM 8. For integers $n \geq 0$ and $k \geq 1$ and a real number $a>0$, we have

$$
\begin{aligned}
\hat{T}_{2, a}^{(0)}(n) & =\sum_{l=0}^{n}(-1)^{n-l} \frac{n !}{l !} \hat{c}_{l}, \\
\hat{T}_{2, a}^{(k)}(n) & =\frac{\hat{T}_{2, a}^{(0)}(n)}{a^{k}}-n \sum_{j=1}^{k} \frac{\hat{c}_{n, a}^{(j)}}{a^{k-j+1}}, \\
\hat{T}_{2, a}^{(-k)}(n) & =a^{k} \hat{T}_{2, a}^{(0)}(n)+n \sum_{j=0}^{k-1} a^{k-j-1} \hat{c}_{n, a}^{(-j)} .
\end{aligned}
$$

Putting $k=1$ in (31), we have the following identity. This is also an analogous formula to Euler's identity (3). If $a=1$, then this is a version of Cauchy numbers of the second kind, compared to the version of the first kind (4).

COROLlary 4.

$$
a \sum_{i=0}^{n}\left(\begin{array}{l}
n \\
i
\end{array}\right) \hat{c}_{i} \hat{c}_{n-i, a}=\sum_{l=0}^{n}(-1)^{n-l} \frac{n !}{l !} \hat{c}_{l}-n \hat{c}_{n, a} \quad(n \geq 0) .
$$

Putting $m=3$ in Theorem 7, then for an integer $k, \hat{T}_{3, a}^{(k)}$ can be expressed as follows. This is an extension of [20, Theorem 11].

THEOREM 9. For integers $n \geq 0, k \geq 1$ and a real number $a>0$, we have

$$
\begin{aligned}
\hat{T}_{3, a}^{(0)}(n) & =\sum_{l=0}^{n}(-1)^{n-l} \frac{n !}{l !} \hat{T}_{2,1}^{(1)}(l) \\
& =(-1)^{n} n ! \sum_{l=0}^{n}\left(\sum_{\lambda=0}^{l}(-1)^{\lambda} \frac{\hat{c}_{\lambda}}{\lambda !}-(-1)^{l} \frac{l \hat{c}_{l}}{l !}\right),
\end{aligned}
$$




$$
\begin{aligned}
\hat{T}_{3, a}^{(k)}(n)= & \frac{\hat{T}_{3, a}^{(0)}(n)}{a^{k}}+n !\left(\frac{1}{a^{k}}-\frac{1}{(a+1)^{k}}\right) \sum_{l=0}^{n-1} \frac{(-1)^{n-l-1}(n-l)}{l !} \hat{c}_{l} \\
& +n(n-1) \sum_{j=1}^{k}\left(\frac{1}{a^{k-j+1}}-\frac{1}{(a+1)^{k-j+1}}\right)\left(\hat{c}_{n, a}^{(j)}+(n-2) ! \sum_{l=0}^{n-2}(-1)^{n-l} \frac{\hat{c}_{l+1, a}^{(j)}}{l !}\right), \\
\hat{T}_{3, a}^{(-k)}(n)= & a^{k} \hat{T}_{3, a}^{(0)}(n)-n !\left((a+1)^{k}-a^{k}\right) \sum_{l=0}^{n-1} \frac{(-1)^{n-l-1}(n-l)}{l !} \hat{c}_{l} \\
& +n(n-1) \sum_{j=0}^{k-2}\left((a+1)^{k-j-1}-a^{k-j-1}\right)\left(\hat{c}_{n, a}^{(-j)}+(n-2) ! \sum_{l=0}^{n-2}(-1)^{n-l} \frac{\hat{c}_{l+1, a}^{(-j)}}{l !}\right) .
\end{aligned}
$$

\section{Two kinds of poly-Cauchy numbers}

Define $U_{m, a}^{(k)}(n)$ by

$$
U_{m, a}^{(k)}(n):=\sum_{\substack{i_{1}+\cdots+i_{m}=n \\
i_{1}, \ldots, i_{m} \geq 0}}\left(\begin{array}{c}
n \\
i_{1}, \ldots, i_{m}
\end{array}\right) \underbrace{c_{i_{1}} \cdots c_{i_{m-1}}}_{m-1} \hat{c}_{i_{m}, a}^{(k)} \quad(m \geq 1, n \geq 0) .
$$

Then we obtain the following. The proof is similar to Theorem 1 and omitted.

THEOREM 10. For an integer $k$ and a non-negative integer $m$, we have

$$
\sum_{l=0}^{m}(-1)^{m-l} f_{m, l} U_{m+1, a}^{(k-l)}(n)= \begin{cases}\sum_{l=0}^{m} \sum_{i=0}^{n-m} \frac{n !}{i !}\left(\begin{array}{c}
l \\
n-m-i
\end{array}\right)\left\{\begin{array}{c}
m \\
l
\end{array}\right\} \hat{c}_{l+i, a}^{(k)} & (n \geq m) ; \\
0 & (0 \leq n \leq m-1) .\end{cases}
$$

Putting $m=1$ in Theorem 10, we have the following. This is also an analogous formula to Euler's identity (3).

\section{COROLlary 5.}

$$
\sum_{i=0}^{n}\left(\begin{array}{l}
n \\
i
\end{array}\right) c_{i}\left(\hat{c}_{n-i, a}^{(k-1)}-a \hat{c}_{n-i, a}^{(k)}\right)=n(n-1) \hat{c}_{n-1, a}^{(k)}+n \hat{c}_{n, a}^{(k)} \quad(n \geq 0) .
$$

For a general $m \geq 2$ we have the following. The proof is similar to that of Theorem 5 and omitted.

THEOREM 11. For integers $n \geq 0$ and $k \geq 1$ and a real number $a>0$, we have

$$
U_{m, a}^{(0)}(n)=U_{m-1,1}^{(1)}(n) \text {, }
$$




$$
\begin{aligned}
U_{m, a}^{(k)}(n)= & \sum_{r=0}^{m-2}\left(\begin{array}{l}
n \\
r
\end{array}\right) \sum_{i=0}^{r}\left(\begin{array}{l}
r \\
i
\end{array}\right) \frac{(-1)^{i}}{(i+a)^{k}} U_{m-r, a}^{(0)}(n-r) \\
& +\frac{(-1)^{m-1} n !}{(n-m+1) !} \sum_{j=1}^{k} \sum_{i=0}^{m-2} \frac{(-1)^{i}}{i !(m-i-2) !(i+a)^{k-j+1}} \sum_{\kappa=0}^{m-1} P_{m, \kappa}(n) \hat{c}_{n-\kappa, a}^{(j)}, \\
U_{m, a}^{(-k)}(n)= & \sum_{r=0}^{m-2}\left(\begin{array}{l}
n \\
r
\end{array}\right) \sum_{i=0}^{r}\left(\begin{array}{l}
r \\
i
\end{array}\right)(-1)^{i}(i+a)^{k} U_{m-r, a}^{(0)}(n-r) \\
& +\frac{n !}{(n-m+1) !} \sum_{j=0}^{k-m+1} \sum_{i=0}^{m-2} \frac{(-1)^{m-i}(i+a)^{k-j-1}}{i !(m-i-2) !} \sum_{\kappa=0}^{m-1} P_{m, \kappa}(n) \hat{c}_{n-\kappa, a}^{(-j)} .
\end{aligned}
$$

where $P_{m, \kappa}(n)(\kappa=0,1, \ldots, m-2, m-1)$ are given as in Theorem 5 .

Putting $m=2$ in Theorem 11, then $U_{2, a}^{(k)}$ can be expressed explicitly. This is an extension of [20, Theorem 15].

THEOREM 12. For integers $n \geq 0$ and $k \geq 1$ and a real number $a>0$, we have

$$
\begin{aligned}
U_{2, a}^{(0)}(n) & =\hat{c}_{n}, \\
U_{2, a}^{(k)}(n) & =\frac{\hat{c}_{n}}{a^{k}}-n \sum_{j=1}^{k} \frac{\hat{c}_{n, a}^{(j)}+(n-1) \hat{c}_{n-1, a}^{(j)}}{a^{k-j+1}}, \\
U_{2, a}^{(-k)}(n) & =a^{k} \hat{c}_{n}+n \sum_{j=0}^{k-1} a^{k-j-1}\left(\hat{c}_{n, a}^{(-j)}+(n-1) \hat{c}_{n-1, a}^{(-j)}\right) .
\end{aligned}
$$

Putting $k=1$ in (37), we have the following identity. If $a=1$, then this is reduced to the alternative identity (2.3) in [25, Theorem 2.4] because $c_{n}=\hat{c}_{n}+n \hat{c}_{n-1}$ by (2.2) in [25, Theorem 2.4].

\section{COROLlary 6.}

$$
a \sum_{i=0}^{n}\left(\begin{array}{l}
n \\
i
\end{array}\right) c_{i} \hat{c}_{n-i, a}=\hat{c}_{n}-n\left(\hat{c}_{n, a}+(n-1) \hat{c}_{n-1, a}\right) \quad(n \geq 1) .
$$

Putting $m=3$ in Theorem 11, then $U_{3, a}^{(k)}$ can be expressed as follows. This is an extension of [20, Theorem 17].

THEOREM 13. For integers $n \geq 0$ and $k \geq 1$ and a real number $a>0$, we have

$$
\begin{aligned}
U_{3, a}^{(0)}(n) & =U_{2,1}^{(1)}(n)=-(n-1)\left(\hat{c}_{n}+n \hat{c}_{n-1}\right) \\
& =-(n-1) c_{n},
\end{aligned}
$$




$$
\begin{aligned}
U_{3, a}^{(k)}(n)= & \frac{U_{3, a}^{(0)}(n)}{a^{k}}+\left(\frac{1}{a^{k}}-\frac{1}{(a+1)^{k}}\right) n \hat{c}_{n-1} \\
& +n(n-1) \sum_{j=1}^{k}\left(\frac{1}{a^{k-j+1}}-\frac{1}{(a+1)^{k-j+1}}\right)\left(\hat{c}_{n, a}^{(j)}+(2 n-3) \hat{c}_{n-1, a}^{(j)}+(n-2)^{2} \hat{c}_{n-2, a}^{(j)}\right), \\
U_{3, a}^{(-k)}(n)= & a^{k} U_{3, a}^{(0)}(n)-\left((a+1)^{k}-a^{k}\right) n \hat{c}_{n-1} \\
& +n(n-1) \sum_{j=0}^{k-2}\left((a+1)^{k-j-1}-a^{k-j-1}\right)\left(\hat{c}_{n, a}^{(-j)}+(2 n-3) \hat{c}_{n-1, a}^{(-j)}+(n-2)^{2} \hat{c}_{n-2, a}^{(-j)}\right) .
\end{aligned}
$$

Define $V_{m, a}^{(k)}(n)$ by

$$
V_{m, a}^{(k)}(n):=\sum_{\substack{i_{1}+\cdots+i_{m}=n \\
i_{1}, \ldots, i_{m} \geq 0}}\left(\begin{array}{c}
n \\
i_{1}, \ldots, i_{m}
\end{array}\right) \underbrace{\hat{c}_{i_{1}} \cdots \hat{c}_{i_{m-1}}}_{m-1} c_{i_{m}, a}^{(k)} \quad(m \geq 1, n \geq 0) .
$$

Then we obtain the following. The proof is similar to Theorem 6 and omitted.

THEOREM 14. For an integer $k$ and a non-negative integer $m$, we have

$$
\begin{aligned}
& \sum_{l=0}^{m}(-1)^{m-l} f_{m, l} V_{m+1, a}^{(k-l)}(n) \\
= & \begin{cases}(-1)^{n-m} \sum_{l=0}^{m-1} \sum_{i=0}^{n-m}(-1)^{i} \frac{n !}{i !}\left(\begin{array}{c}
n-l-i-1 \\
n-m-i
\end{array}\right)\left\{\begin{array}{c}
m \\
l
\end{array}\right\} c_{l+i}^{(k)}+\frac{n !}{(n-m) !} c_{n}^{(k)} & (n \geq m) ; \\
0 & (0 \leq n \leq m-1) .\end{cases}
\end{aligned}
$$

Putting $m=1$ in Theorem 14, we have the following.

\section{COROLlary 7.}

$$
\sum_{i=0}^{n}\left(\begin{array}{l}
n \\
i
\end{array}\right) \hat{c}_{i}\left(c_{n-i, a}^{(k-1)}-a c_{n-i, a}^{(k)}\right)=n c_{n, a}^{(k)} \quad(n \geq 0) .
$$

For a general $m \geq 2$ we have the following. The proof is similar to that of Theorem 7 and omitted.

THEOREM 15. For integers $n \geq 0$ and $k \geq 1$ and a real number $a>0$, we have

$$
\begin{aligned}
& V_{m, a}^{(0)}(n)=V_{m-1,1}^{(1)}(n) \\
& V_{m, a}^{(k)}(n)=\frac{V_{m, a}^{(0)}(n)}{a^{k}}+\sum_{r=1}^{m-2} \frac{1}{r !} \sum_{i=0}^{r}\left(\begin{array}{l}
r \\
i
\end{array}\right) \frac{(-1)^{i+n}}{(i+a)^{k}} \sum_{l=0}^{n-r}\left(\begin{array}{c}
n-l-1 \\
r-1
\end{array}\right)(-1)^{l} \frac{n !}{l !} V_{m-r, a}^{(0)}(l)
\end{aligned}
$$




$$
\begin{aligned}
& +(-1)^{m-1} n ! \sum_{j=1}^{k} \sum_{i=0}^{m-2} \frac{(-1)^{i}}{i !(m-i-2) !(i+a)^{k-j+1}} \\
& \times\left(\frac{\hat{c}_{n, a}^{(j)}}{(n-m+1) !}+\sum_{t=1}^{m-2}\left\{\begin{array}{c}
m-1 \\
m-t-1
\end{array}\right\} \sum_{l=0}^{n-m+1}(-1)^{n-m-l+1}\left(\begin{array}{c}
n+t-m-l \\
t-1
\end{array}\right) \frac{\hat{c}_{m+l-t-1, a}^{(j)}}{l !}\right), \\
& V_{m, a}^{(-k)}(n)=a^{k} V_{m, a}^{(0)}(n)+\sum_{r=1}^{m-2} \frac{1}{r !} \sum_{i=0}^{r}\left(\begin{array}{c}
r \\
i
\end{array}\right)(-1)^{i+n}(i+a)^{k} \sum_{l=0}^{n-r}\left(\begin{array}{c}
n-l-1 \\
r-1
\end{array}\right)(-1)^{l} \frac{n !}{l !} V_{m-r, a}^{(0)}(l) \\
& +n ! \sum_{j=0}^{k-m+1} \sum_{i=0}^{m-2} \frac{(-1)^{m-i}(i+a)^{k-j-1}}{i !(m-i-2) !} \\
& \quad \times\left(\frac{\hat{c}_{n, a}^{(-j)}}{(n-m+1) !}+\sum_{t=1}^{m-2}\left\{\begin{array}{c}
m-1 \\
m-t-1
\end{array}\right\} \sum_{l=0}^{n-m+1}(-1)^{n-m-l+1}\left(\begin{array}{c}
n+t-m-l \\
t-1
\end{array}\right) \frac{\hat{c}_{m+l-t-1, a}^{(-j)}}{l !}\right) .
\end{aligned}
$$

Putting $m=2$ in Theorem 15, then $V_{2, a}^{(k)}$ can be expressed explicitly. This is an extension of [20, Theorem 20].

THEOREM 16. For integers $n \geq 0$ and $k \geq 1$ and a real number $a>0$, we have

$$
\begin{aligned}
V_{2, a}^{(0)}(n) & =c_{n}, \\
V_{2, a}^{(k)}(n) & =\frac{c_{n}}{a^{k}}-n \sum_{j=1}^{k} \frac{c_{n, a}^{(j)}}{a^{k-j+1}}, \\
V_{2, a}^{(-k)}(n) & =a^{k} c_{n}+n \sum_{j=0}^{k-1} a^{k-j-1} c_{n, a}^{(-j)} .
\end{aligned}
$$

Putting $k=1$ in (43), we have the identity (2.3) in [25, Theorem 2.4].

COROLlary 8 .

$$
a \sum_{i=0}^{n}\left(\begin{array}{l}
n \\
i
\end{array}\right) \hat{c}_{i} c_{n-i, a}=c_{n}-n c_{n, a} \quad(n \geq 1) .
$$

Putting $m=3$ in Theorem 15, then $V_{3, a}^{(k)}$ can be expressed as follows. This is an extension of [20, Theorem 22]. Notice that

$$
\hat{c}_{n}=\sum_{l=0}^{n}(-1)^{n-l} \frac{n !}{l !} c_{l}
$$


THEOREM 17. For integers $n \geq 0$ and $k \geq 1$ and a real number $a>0$, we have

$$
\begin{aligned}
V_{3, a}^{(0)}(n)= & V_{2,1}^{(1)}(n)=-(n-1) c_{n}, \\
V_{3, a}^{(k)}(n)= & \frac{V_{3, a}^{(0)}(n)}{a^{k}}-\left(\frac{1}{a^{k}}-\frac{1}{(a+1)^{k}}\right) n \hat{c}_{n-1} \\
& +n(n-1) \sum_{j=1}^{k}\left(\frac{1}{a^{k-j+1}}-\frac{1}{(a+1)^{k-j+1}}\right)\left(c_{n, a}^{(j)}+(n-2) ! \sum_{l=0}^{n-2}(-1)^{n-l} \frac{c_{l+1, a}^{(j)}}{l !}\right), \\
V_{3, a}^{(-k)}(n)= & a^{k} V_{3, a}^{(0)}(n)+\left((a+1)^{k}-a^{k}\right) n \hat{c}_{n-1} \\
& +n(n-1) \sum_{j=0}^{k-2}\left((a+1)^{k-j-1}-a^{k-j-1}\right)\left(c_{n, a}^{(-j)}+(n-2) ! \sum_{l=0}^{n-2}(-1)^{n-l} \frac{c_{l+1, a}^{(-j)}}{l !}\right) .
\end{aligned}
$$

\section{References}

[ 1 ] T. AgOH and K. Dilcher, Convolution identities and lacunary recurrences for Bernoulli numbers, J. Number Theory 124 (2007), 105-122.

[2] T. AgOH and K. Dilcher, Higher-order recurrences for Bernoulli numbers, J. Number Theory 129 (2009), 1837-1847.

[ 3 ] T. AGOH and K. Dilcher, Shortened recurrence relations for Bernoulli numbers, Discrete Math. 309 (2009), 887-898.

[4] T. AgOH and K. Dilcher, Recurrence relations for Nörlund numbers and Bernoulli numbers of the second kind, Fibonacci Quart. 48 (2010), 4-12.

[ 5 ] A. BAYAD and Y. HAMAhatA, Polylogarithms and poly-Bernoulli polynomials, Kyushu J. Math. 65 (2011), 15-24.

[6] A. BAYAD and T. KIM, Higher recurrences for Apostol-Bernoulli-Euler numbers, Russ. J. Math. Phys. 19 (2012), 1-10.

[ 7 ] P. F. BYRD, New relations between Fibonacci and Bernoulli numbers, Fibonacci Quart. 13 (1975), 59-69.

[ 8 ] G.-S. Cheon, S.-G. Hwang and S.-G. LeE, Several polynomials associated with the harmonic numbers, Discrete Appl. Math. 155 (2007), 2573-2584.

[ 9 ] L. COMtet, Advanced Combinatorics, Reidel, Dordrecht, 1974.

[10] M.-A. Coppo and B. CAndelpergher, The Arakawa-Kaneko zeta functions, Ramanujan J. 22 (2010), $153-162$.

[11] K. DilcheR, Sums of products of Bernoulli numbers, J. Number Theory 60 (1996), $23-41$.

[12] M. EIE, A note on Bernoulli numbers and Shintani generalized Bernoulli polynomials, Trans. Amer. Math. Soc. 348 (1996), 1117-1136.

[13] R. L. Graham, D. E. Knuth and O. Patashnik, Concrete Mathematics, Second Edition, Addison-Wesley, Reading, 1994.

[14] K. Kamano, Sums of products of Bernoulli numbers, including poly-Bernoulli numbers, J. Integer Seq. 13 (2010), Article 10.5.2.

[15] K. Kamano, Sums of products of hypergeometric Bernoulli numbers, J. Number Theory 130 (2010), 22592271.

[16] K. Kamano and T. Komatsu, Poly-Cauchy polynomials, Mosc. J. Comb. Number Theory 3 (2013), 181207.

[17] M. KaneKo, Poly-Bernoulli numbers, J. Th. Nombres Bordeaux 9 (1997), 221-228. 
[18] T. Komatsu, Poly-Cauchy numbers, Kyushu J. Math. 67 (2013), 143-153.

[19] T. Komatsu, Hypergeometric Cauchy numbers, International J. Number Theory 9 (2013), 545-560.

[20] T. Komatsu, Sums of products of Cauchy numbers, including poly-Cauchy numbers, J. Discrete Math. 2013 (2013), Article ID 373927, (10 pages).

[21] T. Komatsu, Explicit formulae for sums of products of Cauchy numbers including poly-Cauchy numbers, RIMS Kokyuroku Bessatsu B46 (2014), 187-203.

[22] T. Komatsu and L. Szalay, Shifted poly-Cauchy numbers, Lith. Math. J. 54 (2014), 166-181.

[23] H.-M. LiU, S.-H. QI and S.-Y DinG, Some recurrence relations for Cauchy numbers of the first kind, J. Integer Seq. 13 (2010), Article 10.3.8.

[24] T. MACHIDE, Sums of products of Kronecker's double series, J. Number Theory 128 (2008), 820-834.

[25] D. Merlini, R. Sprugnoli and M. C. Verri, The Cauchy numbers, Discrete Math. 306 (2006), 19061920.

[26] A. Petojević, New sums of products of Bernoulli numbers, Integral Transforms Spec. Funct. 19 (2008), 105-114.

[27] A. Petojević and H. M. SRivastava, Computation of Euler's type sums of the products of Bernoulli numbers, Appl. Math. Lett. 22 (2009), 769-801.

[28] W. WANG, Generalized higher order Bernoulli number pairs and generalized Stirling number pairs, J. Math. Anal. Appl. 364 (2010), 255-274.

[29] P. T. YounG, A 2-adic formula for Bernoulli numbers of the second kind and for the Nörlund numbers, J. Number Theory 128 (2008), 2951-2962.

[30] F.-Z. ZHAO, Sums of products of Cauchy numbers, Discrete Math. 309 (2009), 3830-3842.

Present Address:

SChOOL OF Mathematics And Statistics,

WUHAN UNIVERSITY,

WUHAN 430072, CHINA.

e-mail:komatsu@whu.edu.cn 